Revista NEP - Núcleo de Estudos Paranaenses, Curitiba, v. 5, n. 2, dez. 2019

\title{
UMA ANÁLISE DAS RELAÇÕES DE PARENTESCO NA POLÍTICA DO MUNICÍPIO DE IPIRANGA, PR
}

\begin{abstract}
Alexandre Gonçalves Batista ${ }^{1}$
RESUMO: O presente artigo realiza uma análise da perpetuação de determinadas famílias no poder do município de Ipiranga, PR. Para tanto foi levantada a lista de prefeitos e vereadores eleitos nos anos 1912 e 1924 (República Velha) e no período pós-redemocratização, ou seja, a partir de 1945. Uma vez identificadas as famílias com maior presença, levantou-se as relações destas com a economia local e outros laços de poder.
\end{abstract}

Palavras-chave: Genealogia, família, Ipiranga.

\section{AN ANALYSIS OF KIND RELATIONSHIPS IN THE POLICY OF IPIRANGA, PR}

ABSTRACT: This paper analyzes the perpetuation of certain families in power in the municipality of Ipiranga, PR. To this end, the list of mayors and city councilors elected in the years 1912 and 1924 (Old Republic) and in the post-redemocratization period, that is, from 1945 onwards, was identified. with the local economy and other power ties.

Keywords: Genealogy, family, Ipiranga.

\section{Introdução}

"Famílias e determinados sobrenomes estão cada vez mais presentes no espaço político, seja na ocupação de cargos eletivos nas diversas esferas de poder, como também em funções de nomeação, na burocracia estatal" (OLIVEIRA, 2017, p.167). No levantamento da revista Congresso em Foco, de julho de 2017, de Edson Sardinha e com entrevistas dos professores Ricardo Costa de Oliveira e José Marciano Monteiro, foi apontado que a presença das atuais famílias políticas no Congresso Nacional é ainda maior do que nas legislaturas anteriores. Pelo menos 319 deputados (62\% do total da Câmara dos Deputados) e 59 senadores ( $73 \%$ do total de representantes do Senado Federal) têm vínculos de parentesco com políticos, de modo que atualmente quase dois terços dos deputados federais e três quartos dos senadores pertencem às

\footnotetext{
${ }^{1}$ Estudante de Graduação em Engenharia Elétrica pela Universidade Tecnológica Federal do Paraná. Contato: alebat@alunos.utfpr.edu.br
} 
famílias políticas, muitas conhecidas nacionalmente há várias décadas e algumas há séculos² no poder legislativo (SARDINHA, 2017). Dessa forma, fica evidente a pertinência de se analisar a presença contínua de certos clãs familiares na política do Paraná, e no caso deste trabalho, em um município do interior do estado, Ipiranga.

Para este fim, foi levantado os resultados das eleições para prefeito e vereador em Ipiranga a partir de 1947 até 2016 no site do Tribunal Regional Eleitoral (TRE), com exceção de 2016 por não constar no site do TRE, os resultados deste ano foram retirados do site do G1. Além desses anos, também foram analisados os resultados de 1912 e 1924. Então foram analisadas quais famílias se mantiveram no poder nesse período, via comparação de sobrenomes e confirmação em registros, além de uma análise da presença dessas famílias no contexto comercial do município.

\section{O município de Ipiranga}

A história do município foi retirada do site da Prefeitura Municipal de Ipiranga.

Por volta de 1850, uma expedição composta por Valeriano Antônio da Silva, Bento da Silva Leiria, Henrique José Fernandes, Manoel Antunes Ribeiro, Generoso Pinto Leal Taques, Floriano Paes de Almeida, Ponciano da Rocha e José dos Santos Martins, guiada por indígenas, atingiu o território onde hoje se situa o Município de Ipiranga, na região dos Campos Gerais, com seus integrantes instalando-se naquele local, construindo casas e cultivando as terras. Nessa ocasião, deram ao lugarejo a denominação de Guarda Velho. Em 1866, o português Joaquim Teixeira Duarte chegou à localidade, onde encontrou um bom número de moradores, ali fixando residência e iniciando a fundação propriamente dita do povoado, que recebeu a denominação de Ipiranga como homenagem ao local onde foi proclamada a Independência do Brasil. O terreno onde Joaquim Teixeira Duarte lançou os fundamentos da povoação, foi doado por Francisco da Silva Leiria, Emídio Martins Padilha, Manoel Pinto de Oliveira e Dona Maria Joaquina de Andrade

\footnotetext{
${ }^{2}$ Como exemplo, tem-se a família do deputado tucano Bonifácio de Andrada, este com 87 anos e no seu décimo mandato, que representa hoje a quinta geração da família, a qual iniciou suas legislaturas em 1821, ainda nas Cortes Portuguesas. Vale lembrar que Bonifácio é descendente do Patriarca da Independência, José Bonifácio de Andrada (SARDINHA, 2017).
} 
Revista NEP - Núcleo de Estudos Paranaenses, Curitiba, v. 5, n. 2, dez. 2019

Em 1867, Teixeira Duarte providenciou a vinda para Ipiranga, do Padre Antônio Pina, ocasião em que teve início a construção da primeira Capela dedicada à padroeira, Nossa Senhora da Imaculada Conceição. A primeira leva de imigrantes poloneses, alemães e holandeses que chegou a Ipiranga era composta de aproximadamente 519 pessoas, as quais fundaram, na região, as colônias Taió, Ivaí e Bom Jardim.

Por acto de 7 de abril de 1890, foi criado um Distrito Policial, com a denominação de Ipiranga, no Termo de Ponta Grossa e em 7 de dezembro de 1894 Ipiranga foi elevado à categoria de Vila e Município, comarca desmembrada do Município de Ponta Grossa.

\section{Dados atuais}

De acordo com o IPARDES (Instituto Paranaense de Desenvolvimento Econômico e Social), Ipiranga possui uma população de 15.092 habitantes em uma área de $927 \mathrm{~km}^{2}$, tendo assim uma densidade populacional de $16,28 \mathrm{hab} / \mathrm{km}^{2}$, e um grau de urbanização de $34,55 \%$. Em comparação, Curitiba possui uma densidade populacional de 4.027,04 hab $/ \mathrm{km}^{2}$ e Ponta Grossa possui 169,39 hab./km², demonstrando que esse é um município pouco povoado. Além disso, de acordo com o Atlas do Desenvolvimento Humano no Brasil, Ipiranga possuía um IDHM de 0,652 (faixa de desenvolvimento médio) com apenas $31,23 \%$ da população com mais de 18 tendo completado o ensino fundamental.

Esses indicadores apontam para um município majoritariamente rural, marcado pelas grandes propriedades de produção agropecuária (foram 79.170 t de soja produzida em 2017 e um rebanho de 39.000 suínos no mesmo ano de acordo com o IPARDES) e por uma população com baixa escolaridade e vivendo em uma situação mediana de vida característica da área rural do Paraná (0,656 de acordo com o Atlas do Desenvolvimento Humano no Brasil).

\section{Apresentação dos dados}

Primeiramente, é apresentada a Tabela 1 com a lista de eleitos em Ipiranga nos anos de 1912 e 1924, período da República Velha com os partidos de cada um entre parênteses.

Ano: Eleitos: 
Revista NEP - Núcleo de Estudos Paranaenses, Curitiba, v. 5, n. 2, dez. 2019 Dossiê Oligarquias do Nordeste no Brasil

ISSN: 2447-5548

\begin{tabular}{|l|l|l|l|}
\hline 1912 & Prefeito: & João Ribeiro de Freitas & \\
\hline & Vereadores: & Delphino Alves Carneiro & Julio Reinaldo de Miranda \\
\hline & & Hildebrando César de Souza Araújo & Nabor Bento de Souza Labo \\
\hline 1924 & Prefeito: & João Baptista Penteado Sobrinho & Angelo Guaruery \\
\hline & Vereadores: & João Baptista Penteado Sobrinho & Luiz de Jesus Corrêa \\
\hline & & Delphino Alves Carneiro & Felipe Estevan da Costa \\
\hline & & Estanislau Cenwiez & João Gonçalves de Chaves \\
\hline
\end{tabular}

Tabela 1: Eleitos em Ipiranga (República Velha)

A seguir, a Tabela 2 apresenta os eleitos em Ipiranga no Período pós-1945.

\begin{tabular}{|c|c|c|c|}
\hline Ano: & Eleitos: & & \\
\hline \multirow[t]{6}{*}{1947} & Prefeito: & Octavio Cyrillo de Oliveira (PSD) & \\
\hline & Vereadores: & Elias Calixto (PSD) & Teodoro Bardal (PSD) \\
\hline & & José Marques Portela (PSD) & Pedro Luiz Correia (PSD) \\
\hline & & Brandisio Bueno da Silva (PSD) & Alcides Colman de Miranda (PSD) \\
\hline & & $\begin{array}{l}\text { Arnaldo Alfredo Buherer } \\
\text { (UDN/PRP/PTB) }\end{array}$ & $\begin{array}{l}\text { José Mendes Neto } \\
\text { (UDN/PRP/PTB) }\end{array}$ \\
\hline & & Galdino Felix da Silva (UDN/PRP/PTB) & \\
\hline \multirow[t]{6}{*}{1951} & Prefeito: & João de Deus Miranda $(\mathrm{AGV})^{3}$ & \\
\hline & Vereadores: & Otavio Cirilo de Oliveira (AGV) & Paulino Ferreira Messias (AGV) \\
\hline & & Horácio Mendes Sobrinho (AGV) & Brandizio Bueno da Silva (AGV) \\
\hline & & Alcides Colman de Miranda (AGV) & Benedito Vaz Ferreira (AGV) \\
\hline & & Christiano Krahch (CBMR) ${ }^{4}$ & José Mendes Neto (CBMR) \\
\hline & & Francisco da Rocha Carneiro (CBMR) & Estanislau Cenoviz (CBMR) \\
\hline \multirow[t]{3}{*}{1955} & Prefeito: & Octavio C. de Oliveira (PSD) & \\
\hline & Vereadores: & João Leonel Denck (PSD) & Hamilton Guarneri (PSD) \\
\hline & & Bernardino J. Vaz (PSD) & Thomaz Henyda (PSD) \\
\hline
\end{tabular}

\footnotetext{
${ }^{3}$ Aliança Getúlio Vargas PTB/PSD

${ }^{4}$ Coligação Bento Munhoz da Rocha
} 
Revista NEP - Núcleo de Estudos Paranaenses, Curitiba, v. 5, n. 2, dez. 2019 Dossiê Oligarquias do Nordeste no Brasil

ISSN: 2447-5548

\begin{tabular}{|c|c|c|c|}
\hline & & José Marques Portela (PSD) & $\begin{array}{l}\text { Pedro Gonçalves da Silva (Aliança } \\
\text { Ipiranga) }\end{array}$ \\
\hline & & Emilio José Blum (Aliança Ipiranga) & $\begin{array}{l}\text { Estanislau S. Kossemba (Aliança } \\
\text { Ipiranga) }\end{array}$ \\
\hline & & Vitório Manosso (Aliança Ipiranga) & \\
\hline 1959 & Prefeito: & Argemiro Chaves Ferreira (CI) ${ }^{5}$ & \\
\hline & Vereadores: & Alberto Safaid (PSD) & Rubens Faix (PSD) \\
\hline & & Elias Calixto (PSD) & Adolfo Antonio Müller (PSD) \\
\hline & & Jair Ferreira Messias (PSD) & Gumercindo Antunes Mendes (CI) \\
\hline & & Emilio José Blum (CI) & Ubaldo Cezar Krutcz (CI) \\
\hline & & Gustavo Adolfo Neiwerh (CI) & \\
\hline 1963 & Prefeito: & José Marques Portella (PTB) & \\
\hline & Vereadores: & Oswaldo Scheiffer Portella (PTB) & Pedro Jucoski (PTB) \\
\hline & & Agenor Chaves Ferreira (PTB) & Ubaldo Cesar Krutsch (PTB) \\
\hline & & Alberto Safraider (PTB) & Waldomiro Clock (UDN/PDC) \\
\hline & & Emilio Rodrigues Carvalho (UDN/PDC) & $\begin{array}{l}\text { Adolfo Antonio Müller } \\
\text { (UDN/PDC) }\end{array}$ \\
\hline & & João Moleta Sobrinho (UDN/PDC) & \\
\hline 1968 & Prefeito: & Nelson Silveira de Macedo (ARENA) & \\
\hline & Vice: & Alberto Safrais (ARENA) & \\
\hline & Vereadores: & Osvaldo Scheifer Portela (ARN) ${ }^{6}$ & Altair José Chaves (ARN) \\
\hline & & José Izaias Blum (ARN) & Octavio Berger (ARN) \\
\hline & & João Tramontin Filho (ARN) & Geraldo Alceu Dalazoana (ARN) \\
\hline & & João Darci dos Santos (ARN) & $\begin{array}{l}\text { Francisco Camargo de Freitas } \\
(\text { ARN) }\end{array}$ \\
\hline & & João Antonio de Oliveira (ARN) & \\
\hline 1972 & Prefeito: & Osvaldo Schelffer Portela (ARENA) & \\
\hline & Vice: & Levy Jansen (ARENA) & \\
\hline & Vereadores: & Antonio Ribeiro dos Santos (ARENA) & Pedro Terezio Carneiro (ARENA) \\
\hline
\end{tabular}

${ }^{5}$ Coligação Ipiranguense UDN/PSP/PRP

${ }^{6}$ Aliança Renovada Nacional 
Revista NEP - Núcleo de Estudos Paranaenses, Curitiba, v. 5, n. 2, dez. 2019 Dossiê Oligarquias do Nordeste no Brasil

ISSN: 2447-5548

\begin{tabular}{|c|c|c|c|}
\hline & & Ernesto Safaid (ARENA) & Haroldo Antonio Vieira (ARENA) \\
\hline & & Tarcisio Basso (ARENA) & Moacyr Araujo Taques (ARENA) \\
\hline & & Altair José Chaves (ARENA) & João Pedro Rodrigues (ARENA) \\
\hline & & Ataricio Lemes Galvão (ARENA) & \\
\hline 1976 & Prefeito: & Arival Tramontim Ferreira (ARENA) & \\
\hline & Vice: & Achiles Dalazoana (ARENA) & \\
\hline & Vereadores: & José Osmar Mendes Carneiro (ARENA) & Amaur Cezar Blum (ARENA) \\
\hline & & Emilio Rodrigues de Carvalho (ARENA) & Jorge de Brito (ARENA) \\
\hline & & Luiz Gomes Silveira (ARENA) & $\begin{array}{l}\text { Vilson Mendes do Amaral } \\
\text { (ARENA) }\end{array}$ \\
\hline & & Pedro Terezio Carneiro (ARENA) & Antonio Elias Manosso (MDB) \\
\hline & & Ivette Abib (MDB) & \\
\hline 1982 & Prefeito: & Pedro Izaias Blum (PDS) & \\
\hline & Vice: & Pedro Terézio Carneiro (PDS) & \\
\hline & Vereadores: & Luiz Carlos Blum (PDS) & Lauro Bahls Araujo (PDS) \\
\hline & & Altair José Chaves (PDS) & João Maria Paes de Almeida (PDS) \\
\hline & & Antonio Ribeiro Blum (PDS) & Nelson Ferreira de Souza (PMDB) \\
\hline & & Braz Arivaldo Dalazoana (PMDB) & $\begin{array}{l}\text { Nivon Carlos Taborda Scheifer } \\
\text { (PMDB) }\end{array}$ \\
\hline & & Valentim Moleta (PMDB) & \\
\hline 1988 & Prefeito: & Ivo Sheifer (PMDB) & \\
\hline & Vice: & Galileu Dalzoto (PMDB) & \\
\hline & Vereadores: & Neiva Izabel Vieira (PMDB) & Ari Garcia dos Santos (PMDB) \\
\hline & & João A. Scheifer (PMDB) & Iria Costa (PMDB) \\
\hline & & Mário D. Canteri (PMDB) & Antonio Ribeiro Blum (PTB) \\
\hline & & Vilson Mendes do Amaral (PDC) & Ilsor Fernando Roth (PDC) \\
\hline & & Lauro B. De Araújo (PL) & \\
\hline 1992 & Prefeito: & Pedro Izaias Blum (PFL) & \\
\hline & Vereadores: & Ilson Fernando Roth (PFL) & $\begin{array}{l}\text { Jackson Carlo Calixto Moreira } \\
\text { (PST) }\end{array}$ \\
\hline & & Wilson Guse (PMDB) & Antonio Ko \\
\hline
\end{tabular}


Revista NEP - Núcleo de Estudos Paranaenses, Curitiba, v. 5, n. 2, dez. 2019 Dossiê Oligarquias do Nordeste no Brasil ISSN: 2447-5548

\begin{tabular}{|c|c|c|c|}
\hline & & Siguardo Wutzeke (PFL) & Ernesto Traut (PFL) \\
\hline & & Luiz Fernando Betinardi (PMDB) & Fausto Grein Taques (PST) \\
\hline & & Amauri Cesas Blum (PFL) & \\
\hline \multirow[t]{6}{*}{1996} & Prefeito: & Roberto Gomes de Lima (PPB) & \\
\hline & Vereadores: & Jaime Fernandes (PDT) & Alderico Camargo Cominesi (PFL) \\
\hline & & Lauro Balhs Araujo (PTB) & Neiva Izabel Vieira (PDT) \\
\hline & & Luiz Fernando Betinardi (PDT) & Miguel Angelo Blum (PFL) \\
\hline & & João Mielke (PSC) & Luiz Fernando Novatzki (PSC) \\
\hline & & Jose Maria de Camargo (PDT) & \\
\hline \multirow[t]{6}{*}{2000} & Prefeito: & $\begin{array}{l}\text { Roberto Gomes de Lima } \\
\text { (PSDB/PSC/PRP/PPS) }\end{array}$ & \\
\hline & Vereadores: & $\begin{array}{l}\text { Paulo Roberto Blum } \\
\text { (PSDB/PSC/PRP/PPS) }\end{array}$ & $\begin{array}{l}\text { Ernesto Denck Filho } \\
\text { (PSDB/PSC/PRP/PPS) }\end{array}$ \\
\hline & & Julio Cesar Scheifer (PDT/PMDB) & $\begin{array}{l}\text { Alderico Camargo Cominesi } \\
\text { (PFL/PTB) }\end{array}$ \\
\hline & & Argeu Grisol (PSDB/PSC/PRP/PPS) & $\begin{array}{l}\text { José Carlos Cardoso } \\
\text { (PSDB/PSC/PRP/PPS) }\end{array}$ \\
\hline & & Luiz Fernando Betinardi (PDT/PMDB) & Luiz Alberto Blum (PFL/PTB) \\
\hline & & Neudes José Lara (PSDB/PSC/PRP/PPS) & \\
\hline \multirow[t]{2}{*}{2004} & Prefeito: & $\begin{array}{l}\text { Luiz Carlos Blum } \\
\text { (PTB/PMDB/PDT/PT/PP/PTN/PFL) }\end{array}$ & \\
\hline & Vereadores: & $\begin{array}{l}\text { Luiz Fernando Betinardi } \\
\text { (PP/PDT/PT/PTB/PMDB) }\end{array}$ & $\begin{array}{l}\text { Paulo Sérgio Oliveira Ferreira } \\
\text { (PP/PDT/PT/PTB/PMDB) }\end{array}$ \\
\hline & & $\begin{array}{l}\text { Izaías Paes de Almeida } \\
\text { (PL/PPS/PRP/PSDB) }\end{array}$ & Altair Boza Correia (PTN/PFL) \\
\hline & & Luiz Alberto Blum (PTN/PFL) & $\begin{array}{l}\text { Adriana Moleta Guimarães } \\
\text { (PP/PDT/PT/PTB/PMDB) }\end{array}$ \\
\hline & & $\begin{array}{l}\text { Douglas Davi Cruz } \\
\text { (PP/PDT/PT/PTB/PMDB) }\end{array}$ & $\begin{array}{l}\text { Elvira Aparecida Mendes } \\
\text { (PL/PPS/PRP/PSDB) }\end{array}$ \\
\hline & & Argeu Grisol (PL/PPS/PRP/PSDB) & \\
\hline 2008 & Prefeito: & Luiz Carlos Blum & \\
\hline
\end{tabular}


Revista NEP - Núcleo de Estudos Paranaenses, Curitiba, v. 5, n. 2, dez. 2019 Dossiê Oligarquias do Nordeste no Brasil

ISSN: 2447-5548

\begin{tabular}{|c|c|c|c|}
\hline & |Vereadores: & Luiz Fernando Betinardi & Julio Cesar Scheifer \\
\hline & & Sonia Mara do Amaral Mancini & Adriana Moleta Guimarães \\
\hline & & Argeu Grisol & Marco Aurelio Manosso \\
\hline & & Paulo Roberto Blum & Alexandre Zacarias Scheiffer \\
\hline & & Edgar Antonio Gasparelo & \\
\hline 2012 & Prefeito: & Roger Eduardo Angelotti Selski (PR) & \\
\hline & Vice: & Antonio Travensoli Neto (PR) & \\
\hline & Vereadores: & Maicon Vinicius Dalazoana (PSC) & Laertes Prestes (PPS) \\
\hline & & Luiz Fernando Betinardi (PDT) & $\begin{array}{l}\text { Dirceu José Vieira da Rosa } \\
\text { (PMDB) }\end{array}$ \\
\hline & & Inri Makerli Carneiro (PV) & Altair Boza Correia (PPS) \\
\hline & & Geraldo Orovoski (DEM) & Tadeu da Costa Freitas (PSL) \\
\hline & & Miguel Angelo Blum (DEM) & \\
\hline 2016 & Prefeito: & Luiz Carlos Blum (PSC) & \\
\hline & Vice: & Alcides Mayer (PSC) & \\
\hline & Vereadores: & Argeu Grisol (PSL) & Maicon Vinicius Dalazoana (PSC) \\
\hline & & Ivonete Gobel Costa (PTB) & Nelson Odilo Lange (PRB) \\
\hline & & João Mielke (DEM) & Roberto Gomes de Lima (PR) \\
\hline & & Julio Cesar Scheifer (PDT) & Sebastião Braga dos Santos (PTC) \\
\hline & & Laertes Prestes (PSD) & \\
\hline
\end{tabular}

Tabela 2. Eleitos em Ipiranga (pós-1945). Fonte: TER, 2019.

O primeiro fato que podemos inferir é a forte presença da família Blum a partir de 1955, tendo indivíduos carregando o sobrenome eleitos todos os anos a partir de então, com exceção de 1963 e 1972, chegando a eleger um prefeito (Pedro Izaias Blum) e dois vereadores (Luiz Carlos Blum e Antonio Ribeiro Blum) no ano de 1982. A família Blum continua com presença no poder do município até hoje, com o prefeito Luiz Carlos Blum ${ }^{7}$. Sua presença política também é denotada pela presença do neto de Luiz, Luiz Carlos Blum Neto ${ }^{8}$, na Secretaria de

\footnotetext{
${ }^{7}$ Luiz Carlos Blum, Filho de Irene Safraider Blum e Paulino Blum, nascido em 01/07/1948 (de acordo com certidão postada por ele em seu Facebook).

${ }^{8}$ Parentesco analisado pelo Facebook na falta de documentos e certidões oficiais.
} 
Meio Ambiente do município, Vera Lucia Florindo Blum (esposa de Luiz) na Secretaria de Assistência Social.

A família presenta presença também no comércio da cidade com o "Grupo Blum" como consta em seu site (http://supermercadoblum.com.br/grupo_blum), possuindo um supermercado, um auto posto, uma loja de material de construção, uma cerealista agro e uma distribuidora de gás. O primeiro comércio do grupo foi fundado em 1970 pelos irmãos Pedro Albari Blum e Luiz Carlos Blum, sendo que o terceiro irmão, Sebastião Edeval Blum, se juntou à administração em 1975 e "Hoje está na 2a Geração: fillhos, genros, noras" como consta no site.

De acordo com o site Socios Brasil, Luiz Carlos Blum é sócio-administrador de 7 empresas, sendo elas as 5 pertencentes ao Grupo Blum (Supermercados Blum Ltda, Transportadora Blum Ltda, Comercial De Cereais Blum Ltda, Total Engenharia Ltda, Auto Posto Blum Ltda), a Paróquia Cristo Redentor De Curitiba na rua Trajano Reis e outra onde hoje é a Comunidade Monte Moriá, comunidade membra da Paróquia Cristo Redentor de Curitiba. Ainda de acordo com o site, essas empresas totalizam um capital social de 600.000 reais (porém a única que tem um capital social declarado é a Total Engenharia) e a primeira sociedade de Luiz Carlos Blum foi firmada em 13 de Novembro de 1970, aos seus 22 anos.

Outro dado interessante é que de acordo com o site CNPJ Consultas, em todas as empresas, com exceção da Total Engenharia e das paróquias, Luiz Carlos Blum tem como sócios Pedro Albari Blum (irmão), Sebastião Edeval Blum (irmão) e Ligia Maria Aguiar Blum (ex-esposa). Na Total Engenharia ele tem como sócios Fernando Alceu Zettel e João Alexandre Camargo Da Silva enquanto que nas paróquias ele é presidente.

\section{Conclusão}

A partir dos dados levantados e das análises realizadas conclui-se que a família Blum possui não só uma forte presença na política do município, como também é dominante na economia local com suas múltiplas empresas administradas por membros da família. Esse contexto é similar ao analisado por VANALI (2016) “A estruturação de empresas em torno de laços familiares é considerada uma condenação ao fracasso pelos estudos tradicionais, mas 
podemos perceber pela breve análise da família Rocha Loures como o espírito patriarcal e as tendências clânicas não se restringem ao ambiente familiar, mas atingem as empresas, o mundo dos negócios". em VANALI (2016), foi concluído que o estudo deveria ser aprofundado na relação das famílias com a política e com os negócios uma vez que essa relação ainda não foi abordada de maneira abrangente. De maneira similar o presente artigo levanta a necessidade de um estudo mais aprofundado dessa relação, uma vez que os dados sobre as famílias dominantes do interior do estado são escassos e os dados históricos de muitos municípios não são de fácil acesso.

\section{Referências}

GOULART, Mônica H. H. S; OLIVEIRA, Ricardo Costa de; MONTEIRO, José Marciano; VANALI, Ana C. Família, parentesco, instituições e poder no Brasil: retomada e atualização de uma agenda de pesquisa. Revista Brasileira de Sociologia, v. 5, n. 11, set/dez. 2017.

SARDINHA, Edson. (2017), "Congresso, um negócio de família”. Congresso em Foco, ano 6, n. 26, pp. 36-46. Disponível em: https://congressoemfoco.uol.com.br/especial/noticias/congresso-um-negocio-de-familia-seisem-cada-dez-parlamentares-tem-parentes-na-politica/>. Acesso em: 11 jun. 2019.

TRE. Resultados de eleições municipais TRE-PR. disponível em: <http://www.trepr.jus.br/eleicoes/resultados/resultados-de-eleicoes-municipais-tre-pr $>$. Acesso em: 07 jun. 2019.

G1. Eleições 2016 Resultado da Apuração Ipiranga, PR. 2016. Disponível em: <http://g1.globo.com/pr/campos-gerais-sul/eleicoes/2016/apuracao/ipiranga.html>. Acesso em: 07 jun. 2019.

PREFEITURA MUNICIPAL DE IPIRANGA. História. Disponível em: <http://ipiranga.pr.gov.br/pagina/78_Historia.html>. Acesso em: 07 jun. 2019.

IPARDES. CADERNO ESTATÍSTICO MUNICÍPIO DE IPIRANGA. Fevereiro de 2019.

PNUD; IPEA; FJP. Atlas do Desenvolvimento Humano no Brasil. Disponível em: <http://atlasbrasil.org.br/2013/pt/perfil_m/ipiranga_pr>. Acesso em: 07 jun. 2019.

Grupo Blum - Historia. Disponível em: 〈http://supermercadoblum.com.br/grupo_blum>. Acesso em: 11 jun. 2019.

SOCIOS BRASIL. 〈http://www.sociosbrasil.com/nome/luiz-carlos-blum>. Acesso em: 2 jul. 2019. 
CNPJ CONSULTAS. <https://www.cnpjconsultas.com>. Acesso em: 2 jul. 2019.

VANALI, Ana C.; CRUZ, Katiano Miguel. Um exemplo de Old Money no Paraná: a família Rocha Loures. Revista NEP, Curitiba, v. 2, n. 2, p. 1-26, 2016.

Observações:

http://www.pmtb.pr.gov.br/noticia.php?codigo=1553 (em 2009 Ligia era a primeira dama de Ipiranga, portanto esposa de Luiz Carlos Blum, porém em 2016 Luiz Carlos é declarado divorciado no site da Gazeta do Povo das eleições ano https://especiais.gazetadopovo.com.br/eleicoes/2016/ipiranga-pr/prefeito/luiz-blum-20/ . Porém de acordo com o facebook de Luiz e de Vera Lúcia Florindo Blum, eles são casados desde de 2012 pelo menos, então é provável que Luiz se divorciou e casou de volta entre 2009 e 2012)

Recebido em: 28 out. 2019

Aceito em: 26 nov. 2019 